\title{
Association between Sub-Threshold Affective Symptoms and Prefrontal Activation in Non-Clinical Population-An NIRS Study
}

\author{
Koichiro Fujimaki1 ${ }^{*}$, Hidenori Takemoto' ${ }^{1}$, Shigeru Morinobu ${ }^{2}$ \\ ${ }^{1}$ Faculty of Health and Welfare, Prefectural University of Hiroshima, Hiroshima, Japan \\ ${ }^{2}$ Department of Psychiatry, Kochi University School of Medicine, Kochi, Japan \\ Email: ${ }^{*}$ ujimaki@pu-hiroshima.ac.jp
}

Received 14 May 2014; revised 12 June 2014; accepted 7 July 2014

Copyright (C) 2014 by authors and Scientific Research Publishing Inc.

This work is licensed under the Creative Commons Attribution International License (CC BY). http://creativecommons.org/licenses/by/4.0/

(c) (i) Open Access

\section{Abstract}

Only a few studies have examined the relationship between self-assessment of affective symptoms and brain activation in a non-clinical population. The aim of the present study was to assess this relationship and examine the underlying cortical mechanisms in a non-clinical population. Seventy-nine healthy volunteers were assessed for affective symptoms using the Zung Self-rating Depression Scale (SDS), for apathy using the Apathy Scale (AS), and for feelings of stress using the Stress Arousal Checklist (SACL). Participants also performed a serial arithmetic task according to the Uchida-Kraepelin performance test while hemoglobin concentration changes were assessed on the surface of the prefrontal cortex (PFC) using 32-channel near-infrared spectroscopy (NIRS). The activity on the right side of PFC had a significant negative correlation with the SDS score. The AS and SACL scores were positively correlated with the SDS score. Furthermore, in a multiple regression analysis, SDS scores were predicted by the activity of the right PFC, AS scores, and SACL scores. These results suggest that the association between the cortical activation changes, apathy, and feelings of stress may objectively identify individuals with sub-threshold affective symptoms.

\section{Keywords}

Sub-Threshold Affective Symptoms, Apathy, Feelings of Stress, Serial Arithmetic Task, Prefrontal Cortex, Near-Infrared Spectroscopy

\footnotetext{
*Corresponding author.
}

How to cite this paper: Fujimaki, K., Takemoto, H., \& Morinobu, S. (2014). Association between Sub-Threshold Affective Symptoms and Prefrontal Activation in Non-Clinical Population-An NIRS Study. Psychology, 5, 1024-1034. 


\section{Introduction}

Variability in affective state has been observed in non-clinical populations, and such variability has been shown to have a high correlation with psychosocial functioning. For example, the presence of sub-syndromal depressive symptoms has a negative impact on psychological functioning (Judd et al., 1996).

Investigation of the relationship between affective symptoms and brain activation is one approach to understanding the underlying nature of affective symptoms in more depth. Many brain activation studies using a combination of brain images and cognitive tasks have helped reveal the neural basis of psychiatric disorders such as major depressive disorder (MDD) (Harvey et al., 2005; Hugdahl et al., 2004; Pu et al., 2008). Interestingly, most studies using a phonemic verbal fluency task (e.g., producing words that begin with a particular letter) showed hypoactivation of the prefrontal cortex in patients with MDD (Audenaert et al., 2002; Matsuo et al., 2002; E. Okada \& Delpy, 2003; Videbech, 2000). In contrast, studies of MDD using other effortful cognitive tasks identified hyperactivation of the prefrontal cortex (PFC), indicating possible compensatory activity to maintain task performance (Harvey et al., 2005; Hugdahl et al., 2004; Matsuo et al., 2007). Although the corresponding author in the present study previously reported that affective symptoms were associated with prefrontal hyperactivation using the Trail Making Test (TMT) in a non-clinical population (Sawa et al., 2012), only a few studies have examined the relationships between affective symptoms, cognitive function, and brain activation in a non-clinical population. Furthermore, it is not clear whether sub-threshold affective symptoms of individual subjects interact with brain activation during a serial arithmetic task.

NIRS has been recently investigated as a noninvasive means of assessing functional activity in the brain via measured hemodynamic responses (Sitaram et al., 2007). Unlike electroencephalograms (EEGs), however, NIRS measurements do not require cumbersome skin preparation and electrode gels. Moreover, the thought processes required to intentionally generate the NIRS signals are relatively simple and more directly reflect cognitive function (Coyle et al., 2004). NIRS has been used for the non-invasive measurement of concentration changes in oxygenated hemoglobin (oxy-Hb), deoxygenated hemoglobin (deoxy-Hb), and total hemoglobin (total-Hb) related to brain functions. Optical topography is an application of NIRS using multiple measurement positions that allow brain activation to be imaged (Maki et al., 1995). Optical topography is uniquely useful because it is non-invasive and can be used without restraining the subject. NIRS determines the properties of brain tissue by transmitting near-infrared electromagnetic radiation (650 - $950 \mathrm{~nm}$ wave lengths) through the skull and comparing the intensities of the returning and incident light. Because the fraction of light absorbed versus the fraction transmitted is dependent on the concentrations of chromophores, NIRS can be used to assess hemodynamic responses in regions such as the motor cortex (using motor imagery tasks (Sitaram et al., 2007)) and the PFC (using music imagery (Blood \& Zatorre, 2001), mental arithmetic (Villringer \& Chance, 1997), or preference tasks (Luu \& Chau, 2009)). In this study, we used NIRS to assess the activity of the PFC of the brain during an intelligence task.

This study aimed to evaluate if PFC activity measured by NIRS might represent a biological assessment of sub-threshold affective symptoms when the affective state is evaluated in non-clinical population. We hypothesized that abnormal cortical activation in a non-clinical population, as measured by NIRS, would be associated with sub-threshold affective symptoms in a neuro-psychological profile. We performed the following study to test this hypothesis directly.

\section{Methods}

\subsection{Subjects}

Seventy-nine healthy volunteers (29 males, 50 females) participated in this study (mean age, $20.84 \pm 0.46$ years; range, 20 - 22 years). All subjects were determined to be right-handed using the Edinburgh Handedness Inventory Scale (Oldfield, 1971). Participants were recruited from the Prefectural University of Hiroshima. Participants were recruited primarily via emails, advertisements in a campus newspaper, and recruitment posters. Participants also had to be able and willing to attend the research study. All participants were students. No subject had a history of neurological disorder, major psychiatric disorder, substance abuse, head injury, or major physical illness, or was using any psychotropic medications at the time of the study. Assessments for affective states were based on self-rating scales with a structured diagnostic interview (Structured Clinical Interview for Diagnostic and Statistical Manual of Mental Disorders, Fourth Edition (DSM-IV)). The study was approved by the 
ethics committee of the Prefectural University of Hiroshima. The content of the study and ethical considerations related to subjects were explained to subjects, and written informed consent to participate in the study was obtained.

\subsection{Activation Task}

The activation task consisted of a 3-min pre-task baseline, a serial arithmetic task, and a 3-min post-task baseline. Each subject sat on a comfortable chair in a quiet room and was ordered to keep his/her head as immobile as possible and not to speak. The task was a serial addition test (Sugimoto et al., 2009) that required subjects to perform calculations as fast and accurately as possible within $15 \mathrm{~min}$. This was achieved using pre-printed paper containing 15 lines of random, single-digit, horizontally aligned numbers. For each minute of the test, the subject was instructed to begin a new line regardless of their position on the current line. Each line contained an excess of calculations such that the subjects were not able to finish any line in a particular minute before being prompted by the examiner to move to the next line for the next minute.

\subsection{Assessment of Intelligence Quotient, Affective Symptoms, Extent of Apathy, and Feelings of Stress}

Each subject was assessed for intelligence quotient (IQ), affective symptoms, extent of apathy, and feelings of stress.

The National Adult Reading Test (NART) was used as simple and quick assessment of intelligence. NART is widely used as a measure of premorbid IQ. A Japanese version of the NART (JART) was used that contained 50 Japanese irregular words, all of which were Kanji (ideographic script) compound words. Reading performance based on JART and IQ as measured by the Wechsler Adult Intelligence Scale-Revised (WAIS-R) was examined in a sample of 100 normal elderly persons. A linear regression equation was obtained in which the observed full-scale IQ was regressed on the reading errors of the JART (Matsuoka et al., 2006).

Subjective affective symptoms were measured using the Zung Self-rating Depression Scale (SDS) (Zung, 1965), a self-rating scale that consists of 20 questions. The SDS score ranges from 20 (best) to 80 (worst), and the average score in a normal control Japanese population was $35.1 \pm 8.0$ (mean \pm SD) (Fukuda \& Kobayashi, 1983). A higher score on the SDS is indicative of a relatively greater degree of affective symptoms.

The extent of apathy was measured using the Apathy Scale (AS), a self-rating scale for assessing the tendency to apathy that consists of 14 questions (Starkstein et al., 1992; Starkstein et al., 1993). This scale is an abridged version of Marin's Apathy Scale (Marin, 1991). The AS score ranges from 0 (best) to 42 (worst), and the average score in a normal control Japanese was $8.7 \pm 6.6$ (mean \pm SD) population (K. Okada et al., 1997). A higher score on this scale is indicative of a relatively greater degree of apathy.

Each participant's mood was evaluated by the Japanese version of the Stress Arousal Checklist (JSACL). The JSACL (Hatta, 1995) was originally developed from the Stress Arousal Checklist (SACL) by Cox and Mackey (Cox \& Mackay, 1985). JSACL is a validated tool for measuring stress and arousal levels. It has been used in a number of contexts, including general practice (French et al., 2001; Heaney et al., 1998; Porter et al., 1985). JSACL consists of 30 adjectives that describe feelings and moods; respondents use a 4-point scale to indicate how accurately each adjective matches their current state. The range of scores is -26 to 28 for stress and -17 to 19 for arousal. A higher score on this scale is indicative of a relatively greater degree of stress (JSACL-ST) or arousal (JSACL-AR).

\subsection{NIRS Measurement}

In this study, changes in the concentration of oxy-Hb and deoxy-Hb were measured using a 32-channel NIRS machine (FOIRE-3000; Shimadzu Co., Kyoto Japan). This imaging technique is designed with three different wave lengths of 780, 805, and $830 \mathrm{~nm}$ to monitor changes in oxy-Hb, deoxy-Hb, and total-Hb, respectively. The distance between the pair of emission and detector probes was $3.0 \mathrm{~cm}$, and it was considered that the machine could measure points at a depth of 2 to $3 \mathrm{~cm}$ from the scalp, that is, the surface of the cerebral cortex (Hock et al., 1997; Toronov et al., 2001). As shown in Figure 1, the probes of the NIRS machine were placed on the subject's bilateral frontal region. Based on the international 10 - 20 system, the lower central edge of each probe was suited above Fpz, along the reference curve T3-Fpz-T4. The 32 channels probably covered the middle and supe- 


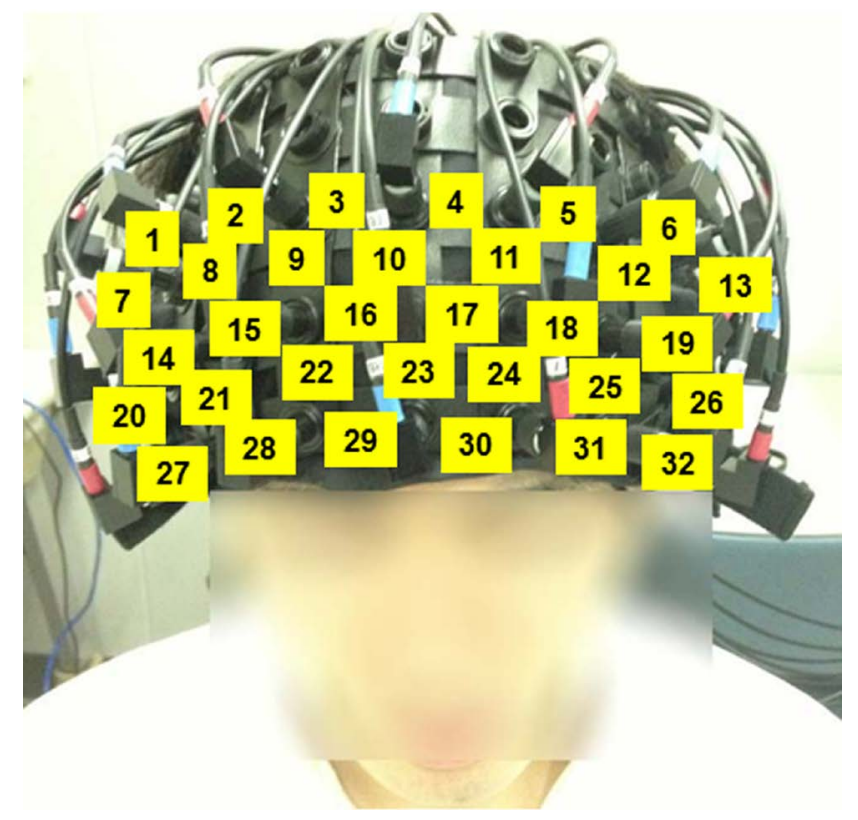

Figure 1. Probe settings and measurement points of the 32channel machine. The probes were placed over the subject's bilateral frontal regions.

rior PFC regions (BA9, 46, 10). The optical signal measured in each trial was transformed into a time series of optical topography signals based on the modified Beer-Lambert law (Maki et al., 1995). We used oxy-Hb signals as the representative optical topography signals in this study, and the time-course data were bandpass-filtered between 0.02 and $0.8 \mathrm{~Hz}$ to remove components originating from the slow fluctuations of cerebral blood flow and heartbeat noise. Smoothing was performed by the moving average method (a boxcar filter) with a sliding time window of $1.1 \mathrm{~s}$.

\subsection{Analytical Methods}

The analysis focused on changes in oxy-Hb, which were defined as the mean concentration of oxy-Hb. Changes in oxy-Hb were assumed to reflect cognitive activation more directly than deoxy-Hb changes, as shown by their stronger correlation with blood-oxygenation level dependent signals measured by functional magnetic resonance imaging (fMRI) (Strangman et al., 2002).

The continuous stream data were divided into blocks that each consisted of a 180-s pre-task period, a 900-s task period, and a 180-s post-task period for further analysis. Before individual averaging, baselines were corrected with mean z-scores of $5 \mathrm{~s}$ before each task. For each subject, the mean change in the concentration of oxy-Hb was calculated for each channel. These measurements were used for statistical analysis. Using the paired t-test, the mean hemoglobin changes were determined. Further, measurement positions with significant mean values were identified as activation positions.

The grand average waveforms of the oxy-Hb concentration changes were obtained for all subjects, based on each subject's waveforms in all 32 channels.

To examine the relationship between the sub-threshold affective symptoms and apathy, the feelings of stress, oxy-Hb changes during the serial arithmetic task, Spearman's rank correlation coefficient was used.

A stepwise multiple regression analysis was conducted to determine the unique contributions of variables assessed (IQ, apathy, the feelings of stress, oxy-Hb changes) to sub-threshold affective symptoms. Then, using variables assessed that showed significant correlations, stepwise multiple regression analysis was done to determine which clinical variables were the best predictors of sub-threshold affective symptoms. Each variable was entered into the multiple regression analysis if its $F$ value was $>4$.

All analytical values were considered significantly different at $p<0.05$. Statistical analyses were performed using PASW Statistics 18.0 software (SPSS Japan Inc., Tokyo, Japan). 


\section{Results}

\subsection{Variables Assessed}

Variables assessed included age, IQ, affective symptoms, extent of apathy, and feelings of stress (Table 1). Data collection of these variables took place from January to March 2013. IQ was assessed by treating psychiatrists. Self-assessment scales were used for the assessment of affective symptoms, apathy, and feelings of stress. These variables were all assessed on the same day. Each variable was assessed a single time.

\subsection{Channels Indicating Cortical Activation}

All channel positions yielded statistical significance indicating cortical activation and reached the $5 \%$ level of statistical significance using t-test.

\subsection{Correlation with $0 x y-H b$ Changes during the Task}

Figure 2 shows the mean of all NIRS data from 79 volunteers during the entire 21-minute test. As shown in Table 2, oxy-Hb changes during the serial arithmetic task were negatively correlated with the SDS score in channel 1 (CH1) $(r=-0.261, p=0.020)$, CH7 ( $r=-0.233, p=0.039)$, and CH14 $(r=-0.233, p=0.039)$. No channel showed oxy-Hb changes during the serial arithmetic task that were positively correlated with the SDS score.

\subsection{Correlation Coefficients between SDS Score and Other Variables Assessed}

As shown in Table 3, AS score and JSACL-ST score were positively correlated with the SDS score (AS: $\mathrm{r}=$ 0.533, $p<0.001$, JSACL-ST: $r=0.513, p<0.001$ ). JSACL-AR was negatively correlated with the SDS score ( $\mathrm{r}$ $=-0.492, p<0.001)$.

\subsection{Stepwise Regression Analysis}

Table 4 shows results of stepwise regression analysis on sub-threshold affective symptoms. SDS was significantly predicted by AS, JSACL-ST, and oxy-Hb changes in $\mathrm{CH} 1$ during the serial arithmetic task.

\section{Discussion}

In this study, we demonstrated that sub-threshold affective symptoms were associated with brain function in a non-clinical population. We showed that participants with a higher score of SDS had a smaller increase in oxy$\mathrm{Hb}$ in the frontal cortical regions in the context of equal performance during the serial arithmetic task. Previous neuroimaging studies on cognitive impairment in patients with MDD demonstrated frontal hypoactivation

Table 1. Subjects’ baseline characteristics.

\begin{tabular}{ccc}
\hline & Mean & SD \\
\hline Participants, no. & 79 & 0.46 \\
Age (years) & 20.8 & 6.2 \\
JART-FIQ & 102.3 & 6.7 \\
JART-VIQ & 103.0 & 4.5 \\
JART-PIQ & 100.9 & 5.9 \\
SDS & 40.0 & 7.1 \\
AS & 14.5 & 8.5 \\
JSACL-ST & -8.6 & 6.5 \\
JSACL-AR & 2.2 & \\
\hline
\end{tabular}

Note: JART = Japanese Adult Reading Test; FIQ = Full Scale Intelligence Quotient; VIQ = Verbal Intelligence Quotient; PIQ = Performance Intelligence Quotient; SDS = Zung Self-rating Depression Scale; AS = Apathy Scale; JSACL = Stress Arousal Check List, Japanese Version; ST = Feelings of Stress; $\mathrm{AR}=$ Feelings of Arousal; SD = Standard Deviation. 


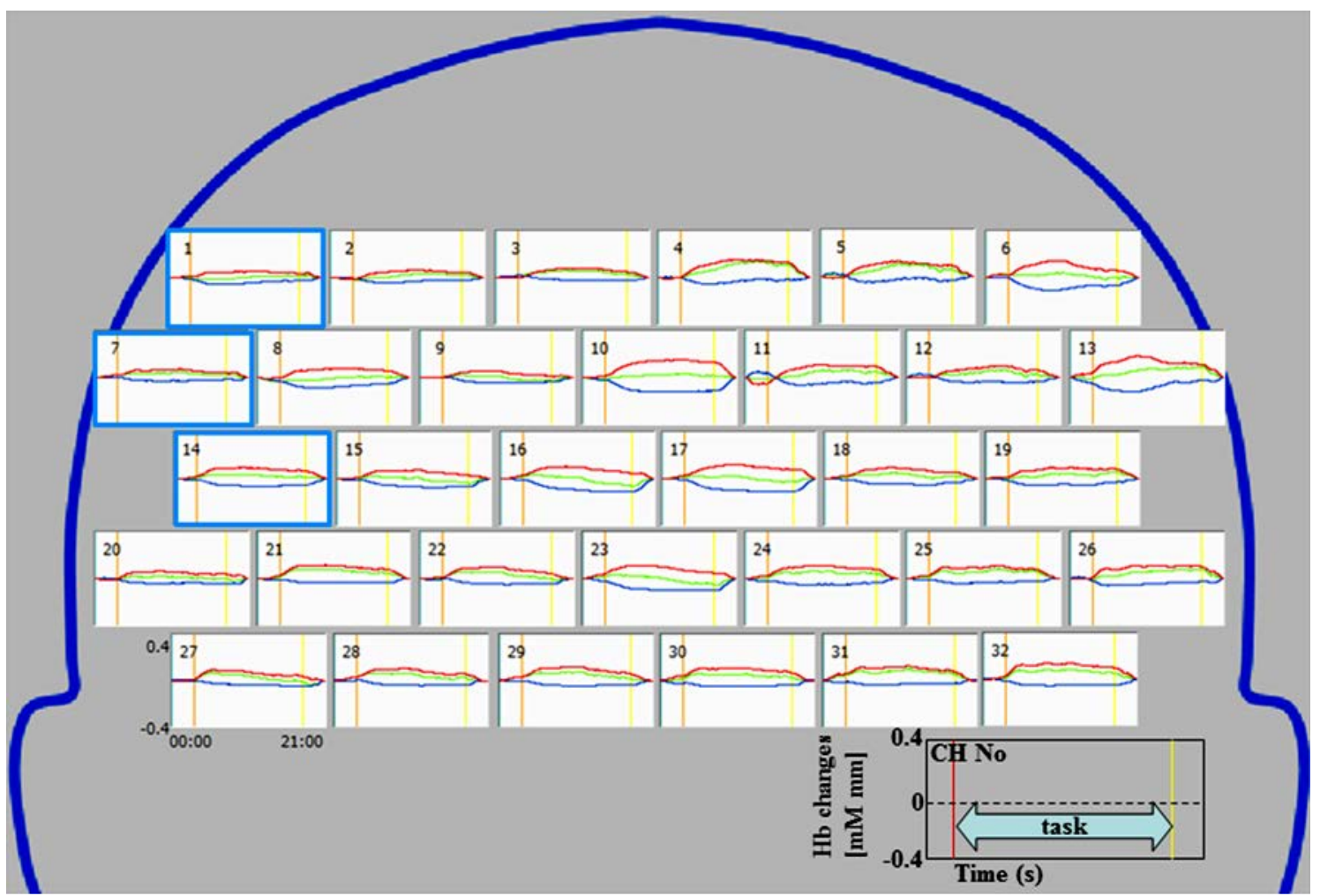

Figure 2. Grand average waveforms of oxygenated, deoxygenated and total hemoglobin concentration changes. Grand average waveforms of oxygenated (red lines), deoxygenated (blue lines) and total (green lines) hemoglobin concentration changes during a 15-min serial arithmetic task (between the red and yellow lines) measured using the 32-channel NIRS machine over the frontal regions. The blue frame indicates the channel showing a significant correlation with the SDS.

Table 2. Correlation coefficients between SDS score and oxy-Hb changes during a serial arithmetic task.

\begin{tabular}{|c|c|c|c|c|c|c|c|}
\hline Channels & CH1 & CH2 & CH3 & CH4 & CH5 & CH6 & \\
\hline & $-0.261^{*}$ & 0.020 & 0.015 & 0.030 & 0.082 & 0.028 & \\
\hline \multirow[t]{2}{*}{ Channels } & CH7 & CH8 & CH9 & CH10 & CH11 & CH12 & CH13 \\
\hline & $-0.233^{*}$ & -0.025 & -0.080 & -0.035 & -0.080 & -0.054 & 0.035 \\
\hline \multirow[t]{2}{*}{ Channels } & CH14 & CH15 & CH16 & CH17 & CH18 & CH19 & \\
\hline & $-0.233^{*}$ & -0.168 & -0.116 & -0.204 & -0.104 & -0.036 & \\
\hline \multirow[t]{2}{*}{ Channels } & CH20 & CH21 & CH22 & CH23 & CH24 & CH25 & CH26 \\
\hline & -0.089 & -0.145 & -0.117 & -0.161 & -0.118 & -0.191 & -0.160 \\
\hline \multirow[t]{2}{*}{ Channels } & CH27 & CH28 & CH29 & СН30 & СН31 & СН32 & \\
\hline & -0.108 & -0.032 & -0.158 & -0.160 & -0.052 & 0.061 & \\
\hline
\end{tabular}

Note: ${ }^{*} p<0.05 . ; \mathrm{CH}=$ Channel.

(Audenaert et al., 2002; Matsuo et al., 2002; E. Okada \& Delpy, 2003; Videbech, 2000) and hyperactivation (Harvey et al., 2005; Hugdahl et al., 2004; Matsuo et al., 2007). Our results are consistent with Matthews' study (Matthews et al., 2009), who reported hypoactivity in the subgenual cingulate during inhibitory processing associated with symptom severity in patients with MDD using fMRI.

We showed that oxy-Hb changes in the frontal regions during the serial arithmetic task were negatively correlated with the SDS score using NIRS. There is no previous study that investigated the relationship between 
Table 3. Correlation coefficients between SDS score and other variables assessed.

\begin{tabular}{cc}
\hline Variables Assessed & SDS \\
\hline JART-FIQ & 0.011 \\
JART-VIQ & 0.010 \\
JART-PIQ & 0.005 \\
AS & $0.533^{*}$ \\
JSACL-ST & $0.513^{*}$ \\
JSACL-AR & $-0.492^{*}$ \\
\hline
\end{tabular}

Note: ${ }^{*} p<0.001$; SDS = Zung Self-rating Depression Scale; JART = Japanese Adult Reading Test; FIQ = Full Scale Intelligence Quotient; VIQ = Verbal Intelligence Quotient; PIQ = Performance Intelligence Quotient; AS = Apathy Scale; JSACL = Stress Arousal Check List, Japanese Version; $\mathrm{ST}=$ Feelings of Stress; AR = Feelings of Arousal.

Table 4. Stepwise regression for SDS score.

\begin{tabular}{cccc}
\hline Dependent variable & Independent variable & Adjusted $\mathbf{R}^{2}$ & $\boldsymbol{\beta}$ \\
\hline SDS & AS & $0.455^{*}$ & $0.421^{*}$ \\
& JSACL-ST & & $0.343^{*}$ \\
CH1 & $-0.256^{*}$ \\
\hline
\end{tabular}

Note: ${ }^{*} p<0.01 . ;$ SDS = Zung Self-rating Depression Scale; AS = Apathy Scale; JSACL = Stress Arousal Check List, Japanese Version; ST = Feelings of Stress; $\mathrm{CH}=$ Channel.

self-assessment of affective symptoms and frontal activation using a serial arithmetic task in non-clinical population. However, some studies have examined this relationship using other cognitive tasks. Previous NIRS studies have consistently shown that MDD patients have less activation in the frontal cortical regions than healthy controls during the verbal fluency task (Herrmann et al., 2004; Kameyama et al., 2006; Matsuo et al., 2000; Matsuo et al., 2002; Ohta et al., 2008; Pu et al., 2008; Suto et al., 2004).

We showed that oxy-Hb changes in the right PFC during the serial arithmetic task were negatively correlated with SDS using NIRS. The present findings have implications for understanding the clinical presentation of individuals with sub-threshold affective symptoms. Recent research suggested that reduced right frontal and temporal cortex activation visible by NIRS during the verbal fluency task is related to the severity of symptoms of MDD (Noda et al., 2012). In addition, the various right hemisphere regions taken together are ideally suited for a threat-response system (Nitschke et al., 2000). Frontally mediated mechanisms inhibit ongoing behavior and interrupt ongoing activity to stop and take stock of a situation. Thus, depression-related activity in right dorsolateral PFC (DLPFC) signals the presence of a threat and is associated with negative affect, avoidance or withdrawal behavior, anxious arousal, and other behavioral manifestations indicative of a threat response (Herrington et al., 2010). In the present study, we placed $3 \times 5$ flexible optode holders on the subject's bilateral frontal region as the lower central edge of each probe was suited above Fpz. Tsuzuki's study demonstrated how to implement the virtual registration method in actual situations. They suggested that, if the lower central edge of each probe is suited above Fpz, the frontopolar column is read as the reference optode on the third row of the third column of the holder, which is placed on Fpz so that the third optode row aligns to the T7-Fpz-T8 reference curve (Tsuzuki et al., 2007). Therefore, the upper side of each probe seems to be nearer to the DLPFC. In the present study, it was suggested that the channels nearer to the right DLPFC were associated with the SDS score. In the present study, oxy-Hb changes during the serial arithmetic task were negatively correlated with the SDS score in $\mathrm{CH} 1$, $\mathrm{CH}$, $\mathrm{CH} 14$; stepwise regression showed that only oxy-Hb changes in $\mathrm{CH} 1$ predicted SDS. $\mathrm{CH} 1$ is the most posterior channel among all channels and in the right side of PFC.

As shown in Table 3, the AS score was positively correlated with the SDS score. Yang's study suggested that apathy should probably be regarded as different from depression and requires distinct prognostic and therapeutic strategies (Yang et al., 2013). For example, it has been found that in patients with a stroke, crying and sadness were associated with a subjective feeling of depression whereas apathy was not (Carota et al., 2005). However, 
in the present study, as a relationship between apathy and depression of sub-syndromal level in healthy subjects, it was suggested that there was a significant overlap between them. Meanwhile, the JSACL-ST score was positively correlated with the SDS score. Hinton's study (Hinton et al., 1991) suggested that SACL assesses aspects of worry and anticipatory fear rather than either a general emotional stress reaction of unease or a compound of the wide range of emotional reactions associated with stress. In other words, they suggested that SACL is highly correlated with Spielberger's State-Trait Anxiety Inventory "state anxiety" scale. Furthermore, Bieling's study showed that the State-Trait Anxiety Inventory total score was more strongly correlated with measures of depression than with those of anxiety (Bieling et al., 1998). The above-mentioned findings can explain our results that showed strong a positive correlation between JSACL-ST score and SDS score. On the other hand, there was a negative correlation between JSACL-AR score and SDS score. JSACL-AR appears to represent wakefulness/ drowsiness or vigor (Cox \& Mackay, 1985). The adjectives belonged to distinct categories: arousers could be either positive (e.g., active, energetic, activated, alert, lively, stimulated) or negative (e.g., drowsy, tired, idle, sluggish, sleepy) (Mackay et al., 1978). The adjectives belonging to JSACL-AR are similar to words for specific statement (I have trouble sleeping at night, I get tired for no reason) in SDS. Therefore, it could be assumed that there was a negative correlation between JSACL-AR score and SDS score.

The corresponding author in the present study previously reported that the SDS score was associated with prefrontal "hyperactivation" during the TMT (Sawa et al., 2012). Our present study showed that the SDS score was associated with prefrontal "hypoactivation" during the serial arithmetic task in a non-clinical population. The reason for this inconsistency is unclear, but one possible explanation is the difference between the activation tasks that may have caused the discrepancies in brain activation. Many authors have suggested that the TMT reflects several cognitive functions, involving visual search, motor speed, and spatial skills (Lezak et al., 2004; Strauss et al., 2006). Conversely, in a recent meta-analysis including 53 fMRI data sets on brain activation during the arithmetic task, Arsalidou and Taylor identified three distinct regions in the PFC that contribute to performance during mental arithmetic (Arsalidou \& Taylor, 2011). It is assumed that the PFC contributes to the arithmetic task more than the TMT.

Our results are consistent with our hypothesis. We found that frontal hypoactivation was associated with selfassessment of affective symptoms in a non-clinical population. Our present methods combining behavioral and NIRS measurements enabled us to detect the effects of sub-threshold affective symptoms on brain function that would be difficult to detect by behavioral output alone.

The reason we inspected the frontopolar cortex in the present study is as follows. The frontopolar cortex (BA10) is thought to have enlarged and become specialized during hominid evolution (Semendeferi et al., 2001). Enlargement and specialization provide a higher level of control to coordinate both ventrolateral and dorsolateral functions to maximize task performance. The frontopolar area is also thought to serve as a "gateway" between the mental life and the external world (i.e., mediates a mechanism that affects bias between attending to the outside world and to our own thoughts) (Burgess et al., 2007). In short, we thought that the oxy-Hb changes during the task performance may be influenced by the mental life because the frontopolar cortex serves as the gateway between affective state and PFC activity responding to performance.

The present study has several limitations. First, the cross-sectional nature of our study precluded a firm conclusion regarding any causal relationship between frontal hypoactivation and sub-threshold affective symptoms. Second, females were included as participants. Women may have potentially influential factors, such as mood fluctuations across the menstrual cycle, and our findings may be influenced by several factors specific to women. Third, age and IQ were controlled with participants in the present study limited to 20- to 22-year-old people. A previous study has shown age-dependent differences in cerebral activation using NIRS (Kameyama et al., 2004); these are factors that can potentially affect brain function. Fourth, power analysis and corrections for multiple comparisons were not conducted in our study as in most previous NIRS studies. It is obvious that our results cannot provide conclusive results in terms of the relationship between the severity of affective symptoms and right PFC activation. Additional studies are needed to replicate our findings, preferably with larger numbers of subjects and corrections for multiple comparisons (although a correction method for multiple comparisons in an NIRS study have not yet been established) to help further address the possibility of a type 1 error. Further studies should take these factors into account. With these limitations in mind, this study provides evidence to support the hypotheses that sub-threshold affective symptoms are associated with abnormal cortical activation during the serial arithmetic task in a non-clinical population. 
In conclusion, the degree of sub-threshold affective symptoms was associated with prefrontal hypoactivation, which is combined with apathy and a high degree of feelings of stress. On the basis of these findings, we assume that the combination of apathy, feelings of stress, and the right side of PFC during the serial arithmetic task measured by NIRS may be used objectively to identify individuals with sub-threshold affective symptoms. Further functional neuroimaging studies focusing on affective states at a non-clinical level may elucidate the brain mechanisms underlying these conditions. These studies may be beneficial for assessment of mental health in healthy subjects and the prevention of suffering from affective symptoms.

\section{References}

Arsalidou, M., \& Taylor, M. J. (2011). Is $2+2=4$ ? Meta-Analyses of Brain Areas Needed for Numbers and Calculations. Neuroimage, 54, 2382-2393. http://dx.doi.org/10.1016/j.neuroimage.2010.10.009

Audenaert, K., Goethals, I., Van Laere, K., Lahorte, P., Brans, B., Versijpt, J., Vervaet, M., Beelaert, L., Van Heeringen, K., \& Dierckx, R. (2002). Spect Neuropsychological Activation Procedure with the Verbal Fluency Test in Attempted Suicide Patients. Nuclear Medicine Communications, 23, 907-916. http://dx.doi.org/10.1097/00006231-200209000-00015

Bieling, P. J., Antony, M. M., \& Swinson, R. P. (1998). The State-Trait Anxiety Inventory, Trait Version: Structure and Content Re-Examined. Behaviour Research and Therapy, 36, 777-788. http://dx.doi.org/10.1016/S0005-7967(98)00023-0

Blood, A. J., \& Zatorre, R. J. (2001). Intensely Pleasurable Responses to Music Correlate with Activity in Brain Regions Implicated in Reward and Emotion. Proceedings of the National Academy of Sciences of the United States of America, 98, 11818-11823. http://dx.doi.org/10.1073/pnas.191355898

Burgess, P. W., Dumontheil, I., \& Gilbert, S. J. (2007). The Gateway Hypothesis of Rostral Prefrontal Cortex (Area 10) Function. Trends in Cognitive Sciences, 11, 290-298. http://dx.doi.org/10.1016/j.tics.2007.05.004

Carota, A., Berney, A., Aybek, S., Iaria, G., Staub, F., Ghika-Schmid, F., Annable, L., Guex, P., \& Bogousslavsky, J. (2005). A Prospective Study of Predictors of Poststroke Depression. Neurology, 64, 428-433.

http://dx.doi.org/10.1212/01.WNL.0000150935.05940.2D

Cox, T., \& Mackay, C. (1985). The Measurement of Self-Reported Stress and Arousal. British Journal of Psychology, 76, 183-186. http://dx.doi.org/10.1111/j.2044-8295.1985.tb01941.x

Coyle, S., Ward, T., Markham, C., \& Mcdarby, G. (2004). On the Suitability of Near-Infrared (NIR) Systems for Next-Generation Brain-Computer Interfaces. Physiological Measurement, 25, 815-822. http://dx.doi.org/10.1088/0967-3334/25/4/003

French, D. P., Mckinley, R. K., \& Hastings, A. (2001). GP Stress and Patient Dissatisfaction with Nights on Call: An Exploratory Study. GP Stress and Patient Satisfaction. Scandinavian Journal of Primary Health Care, 19, 170-173. http://dx.doi.org/10.1080/028134301316982397

Fukuda, K., \& Kobayashi, S. (1983). SDS Manual, Japanese Version (Nihon-Ban SDS Shiyou-Tebiki). Kyoto: Sankyodo.

Harvey, P. O., Fossati, P., Pochon, J. B., Levy, R., Lebastard, G., Lehericy, S., Allilaire, J. F., \& Dubois, B. (2005). Cognitive Control and Brain Resources in Major Depression: An fMRI Study Using the N-Back Task. Neuroimage, 26, 860-869. http://dx.doi.org/10.1016/j.neuroimage.2005.02.048

Hatta, T. (1995). Japanese Stress Arousal Checklist: Stress Measurement by Adjective Words. Osaka: Nihon-Igaku.

Heaney, D., Gorman, D., \& Porter, M. (1998). Self-Recorded Stress Levels for General Practitioners Before and After Forming an Out-of-Hours Primary Care Centre. The British Journal of General Practice: The Journal of the Royal College of General Practitioners, 48, 1077-1078.

Herrington, J. D., Heller, W., Mohanty, A., Engels, A. S., Banich, M. T., Webb, A. G., \& Miller, G. A. (2010). Localization of Asymmetric Brain Function in Emotion and Depression. Psychophysiology, 47, 442-454. http://dx.doi.org/10.1111/j.1469-8986.2009.00958.x

Herrmann, M. J., Ehlis, A. C., \& Fallgatter, A. J. (2004). Bilaterally Reduced Frontal Activation during a Verbal Fluency Task in Depressed Patients as Measured by Near-Infrared Spectroscopy. The Journal of Neuropsychiatry and Clinical Neurosciences, 16, 170-175. http://dx.doi.org/10.1176/appi.neuropsych.16.2.170

Hinton, J. W., Rotheiler, E., \& Howard, A. (1991). Confusion between Stress and State Anxiety in a Much Used Self-Report “Stress” Inventory. Personality and Individual Differences, 12, 91-94. http://dx.doi.org/10.1016/0191-8869(91)90136-Y

Hock, C., Villringer, K., Muller-Spahn, F., Wenzel, R., Heekeren, H., Schuh-Hofer, S., Hofmann, M., Minoshima, S., Schwaiger, M., Dirnagl, U., \& Villringer, A. (1997). Decrease in Parietal Cerebral Hemoglobin Oxygenation during Performance of a Verbal Fluency Task in Patients with Alzheimer's Disease Monitored by Means of Near-Infrared Spectroscopy (Nirs)—Correlation with Simultaneous Rcbf-Pet Measurements. Brain Research, 755, 293-303.

http://dx.doi.org/10.1016/S0006-8993(97)00122-4 
Hugdahl, K., Rund, B. R., Lund, A., Asbjornsen, A., Egeland, J., Ersland, L., Landro, N. I., Roness, A., Stordal, K. I., Sundet, K., \& Thomsen, T. (2004). Brain Activation Measured with fMRI During a Mental Arithmetic Task in Schizophrenia and Major Depression. The American Journal of Psychiatry, 161, 286-293. http://dx.doi.org/10.1176/appi.ajp.161.2.286

Judd, L. L., Paulus, M. P., Wells, K. B., \& Rapaport, M. H. (1996). Socioeconomic Burden of Subsyndromal Depressive Symptoms and Major Depression in a Sample of the General Population. The American Journal of Psychiatry, 153, 1411-1417.

Kameyama, M., Fukuda, M., Uehara, T., \& Mikuni, M. (2004). Sex and Age Dependencies of Cerebral Blood Volume Changes during Cognitive Activation: A Multichannel Near-Infrared Spectroscopy Study. Neuroimage, 22, 1715-1721. http://dx.doi.org/10.1016/j.neuroimage.2004.03.050

Kameyama, M., Fukuda, M., Yamagishi, Y., Sato, T., Uehara, T., Ito, M., Suto, T., \& Mikuni, M. (2006). Frontal Lobe Function in Bipolar Disorder: A Multichannel Near-Infrared Spectroscopy Study. Neuroimage, 29, 172-184. http://dx.doi.org/10.1016/j.neuroimage.2005.07.025

Lezak, M. D., Howieson, D. B., Erin, D. B., \& Tranel, D. (2004). Neuropsychological Assessment (4th Ed.). New York: Oxford University Press.

Luu, S., \& Chau, T. (2009). Decoding Subjective Preference from Single-Trial Near-Infrared Spectroscopy Signals. Journal of Neural Engineering, 6, Article ID: 016003. http://dx.doi.org/10.1088/1741-2560/6/1/016003

Mackay, C., Cox, T., Burrows, G., \& Lazzerini, T. (1978). An Inventory for the Measurement of Self-Reported Stress and Arousal. The British Journal of Social and Clinical Psychology, 17, 283-284. http://dx.doi.org/10.1111/j.2044-8260.1978.tb00280.X

Maki, A., Yamashita, Y., Ito, Y., Watanabe, E., Mayanagi, Y., \& Koizumi, H. (1995). Spatial and Temporal Analysis of Human Motor Activity using Noninvasive Nir Topography. Medical Physics, 22, 1997-2005. http://dx.doi.org/10.1118/1.597496

Marin, R. S. (1991). Apathy: A Neuropsychiatric Syndrome. The Journal of Neuropsychiatry and Clinical Neurosciences, 3, 243-254.

Matsuo, K., Kato, T., Fukuda, M., \& Kato, N. (2000). Alteration of Hemoglobin Oxygenation in the Frontal Region in Elderly Depressed Patients as Measured by Near-Infrared Spectroscopy. The Journal of Neuropsychiatry and Clinical Neurosciences, 12, 465-471. http://dx.doi.org/10.1176/appi.neuropsych.12.4.465

Matsuo, K., Kato, N., \& Kato, T. (2002). Decreased Cerebral Haemodynamic Response to Cognitive and Physiological Tasks in Mood Disorders as Shown by Near-Infrared Spectroscopy. Psychological Medicine, 32, 1029-1037. http://dx.doi.org/10.1017/S0033291702005974

Matsuo, K., Glahn, D. C., Peluso, M. A., Hatch, J. P., Monkul, E. S., Najt, P., Sanches, M., Zamarripa, F., Li, J., Lancaster, J. L., Fox, P. T., Gao, J. H., \& Soares, J. C. (2007). Prefrontal Hyperactivation during Working Memory Task in Untreated Individuals with Major Depressive Disorder. Molecular Psychiatry, 12, 158-166. http://dx.doi.org/10.1038/sj.mp.4001894

Matsuoka, K., Uno, M., Kasai, K., Koyama, K., \& Kim, Y. (2006). Estimation of Premorbid IQ in Individuals with Alzheimer's Disease Using Japanese Ideographic Script (Kanji) Compound Words: Japanese Version of National Adult Reading Test. Psychiatry and Clinical Neurosciences, 60, 332-339. http://dx.doi.org/10.1111/j.1440-1819.2006.01510.x

Matthews, S., Simmons, A., Strigo, I., Gianaros, P., Yang, T., \& Paulus, M. (2009). Inhibition-Related Activity in Subgenual Cingulate Is Associated with Symptom Severity in Major Depression. Psychiatry Research, 172, 1-6. http://dx.doi.org/10.1016/j.pscychresns.2008.08.006

Nitschke, J., Heller, W., \& Miller, G. (2000). Anxiety, Stress, and Cortical Brain Function. In J. C. Borod (Ed.), The Neuropsychology of Emotion, Series in Affective Science (pp. 298-319). New York: Oxford University Press.

Noda, T., Yoshida, S., Matsuda, T., Okamoto, N., Sakamoto, K., Koseki, S., Numachi, Y., Matsushima, E., Kunugi, H., \& Higuchi, T. (2012). Frontal and Right Temporal Activations Correlate Negatively with Depression Severity during Verbal Fluency Task: A Multi-Channel Near-Infrared Spectroscopy Study. Journal of Psychiatric Research, 46, 905-912. http://dx.doi.org/10.1016/j.jpsychires.2012.04.001

Ohta, H., Yamagata, B., Tomioka, H., Takahashi, T., Yano, M., Nakagome, K., \& Mimura, M. (2008). Hypofrontality in Panic Disorder and Major Depressive Disorder Assessed by Multi-Channel Near-Infrared Spectroscopy. Depression and Anxiety, 25, 1053-1059. http://dx.doi.org/10.1002/da.20463

Okada, E., \& Delpy, D. T. (2003). Near-Infrared Light Propagation in an Adult Head Model. II. Effect of Superficial Tissue Thickness on the Sensitivity of the Near-Infrared Spectroscopy Signal. Applied Optics, 42, 2915-2922. http://dx.doi.org/10.1364/AO.42.002915

Okada, K., Kobayashi, S., Yamagata, S., Takahashi, K., \& Yamaguchi, S. (1997). Poststroke Apathy and Regional Cerebral Blood Flow. Stroke: A Journal of Cerebral Circulation, 28, 2437-2441. http://dx.doi.org/10.1161/01.STR.28.12.2437

Oldfield, R. C. (1971). The Assessment and Analysis of Handedness: The Edinburgh Inventory. Neuropsychologia, 9, 97-113. http://dx.doi.org/10.1016/0028-3932(71)90067-4 
Porter, A. M., Howie, J. G., \& Levinson, A. (1985). Measurement of Stress as It Affects the Work of the General Practitioner. Family Practice, 2, 136-146. http://dx.doi.org/10.1093/fampra/2.3.136

Pu, S., Matsumura, H., Yamada, T., Ikezawa, S., Mitani, H., Adachi, A., \& Nakagome, K. (2008). Reduced Frontopolar Activation during Verbal Fluency Task Associated with Poor Social Functioning in Late-Onset Major Depression: MultiChannel Near-Infrared Spectroscopy Study. Psychiatry and Clinical Neurosciences, 62, 728-737. http://dx.doi.org/10.1111/j.1440-1819.2008.01882.x

Sawa, M., Yamashita, H., Fujimaki, K., Okada, G., Takahashi, T., \& Yamawaki, S. (2012). Depressive Symptoms and Apathy Are Associated with Psychomotor Slowness and Frontal Activation. European Archives of Psychiatry and Clinical Neuroscience, 262, 493-499. http://dx.doi.org/10.1007/s00406-012-0296-9

Semendeferi, K., Armstrong, E., Schleicher, A., Zilles, K., \& Van Hoesen, G. W. (2001). Prefrontal Cortex in Humans and Apes: A Comparative Study of Area 10. American Journal of Physical Anthropology, 114, 224-241. http://dx.doi.org/10.1002/1096-8644(200103)114:3<224::AID-AJPA1022>3.0.CO;2-I

Sitaram, R., Zhang, H., Guan, C., Thulasidas, M., Hoshi, Y., Ishikawa, A., Shimizu, K., \& Birbaumer, N. (2007). Temporal Classification of Multichannel Near-Infrared Spectroscopy Signals of Motor Imagery for Developing a Brain-Computer Interface. Neuroimage, 34, 1416-1427. http://dx.doi.org/10.1016/j.neuroimage.2006.11.005

Starkstein, S. E., Mayberg, H. S., Preziosi, T. J., Andrezejewski, P., Leiguarda, R., \& Robinson, R. G. (1992). Reliability, Validity, and Clinical Correlates of Apathy in Parkinson's Disease. The Journal of Neuropsychiatry and Clinical Neurosciences, 4, 134-139.

Starkstein, S. E., Fedoroff, J. P., Price, T. R., Leiguarda, R., \& Robinson, R. G. (1993). Apathy Following Cerebrovascular Lesions. Stroke: A Journal of Cerebral Circulation, 24, 1625-1630. http://dx.doi.org/10.1161/01.STR.24.11.1625

Strangman, G., Culver, J. P., Thompson, J. H., \& Boas, D. A. (2002). A Quantitative Comparison of Simultaneous Bold fMRI and NIRS Recordings during Functional Brain Activation. Neuroimage, 17, 719-731.

http://dx.doi.org/10.1006/nimg.2002.1227

Strauss, E., Sherman, E. M. S., \& Spreen, O. (2006). A Compendium of Neuropsychological Tests: Administration, Norms, and Commentary. New York: Oxford University Press.

Sugimoto, K., Kanai, A., \& Shoji, N. (2009). The Effectiveness of the Uchida-Kraepelin Test for Psychological Stress: An Analysis of Plasma and Salivary Stress Substances. Biopsychosocial Medicine, 3, 5. http://dx.doi.org/10.1186/1751-0759-3-5

Suto, T., Fukuda, M., Ito, M., Uehara, T., \& Mikuni, M. (2004). Multichannel Near-Infrared Spectroscopy in Depression and Schizophrenia: Cognitive Brain Activation Study. Biological Psychiatry, 55, 501-511. http://dx.doi.org/10.1016/j.biopsych.2003.09.008

Toronov, V., Webb, A., Choi, J. H., Wolf, M., Michalos, A., Gratton, E., \& Hueber, D. (2001). Investigation of Human Brain Hemodynamics by Simultaneous Near-Infrared Spectroscopy and Functional Magnetic Resonance Imaging. Medical Physics, 28, 521-527. http://dx.doi.org/10.1118/1.1354627

Tsuzuki, D., Jurcak, V., Singh, A. K., Okamoto, M., Watanabe, E., \& Dan, I. (2007). Virtual Spatial Registration of Stand-Alone fNIRS Data to MNI Space. Neuroimage, 34, 1506-1518. http://dx.doi.org/10.1016/j.neuroimage.2006.10.043

Videbech, P. (2000). PET Measurements of Brain Glucose Metabolism and Blood Flow in Major Depressive Disorder: A Critical Review. Acta Psychiatrica Scandinavica, 101, 11-20. http://dx.doi.org/10.1034/j.1600-0447.2000.101001011.x

Villringer, A., \& Chance, B. (1997). Non-Invasive Optical Spectroscopy and Imaging of Human Brain Function. Trends in Neurosciences, 20, 435-442. http://dx.doi.org/10.1016/S0166-2236(97)01132-6

Yang, S. R., Hua, P., Shang, X. Y., Hu, R., Mo, X. E., \& Pan, X. P. (2013). Predictors of Early Post Ischemic Stroke Apathy and Depression: A Cross-Sectional Study. BMC Psychiatry, 13, 164. http://dx.doi.org/10.1186/1471-244X-13-164

Zung, W. W. (1965). A Self-Rating Depression Scale. JAMA Psychiatry, 12, 63-70. http://dx.doi.org/10.1001/archpsyc.1965.01720310065008 
Scientific Research Publishing (SCIRP) is one of the largest Open Access journal publishers. It is currently publishing more than 200 open access, online, peer-reviewed journals covering a wide range of academic disciplines. SCIRP serves the worldwide academic communities and contributes to the progress and application of science with its publication.

Other selected journals from SCIRP are listed as below. Submit your manuscript to us via either submit@scirp.org or Online Submission Portal.
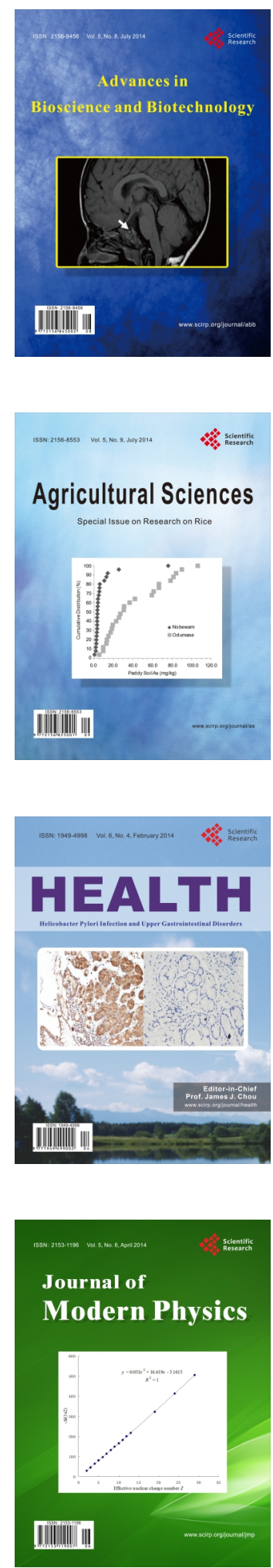
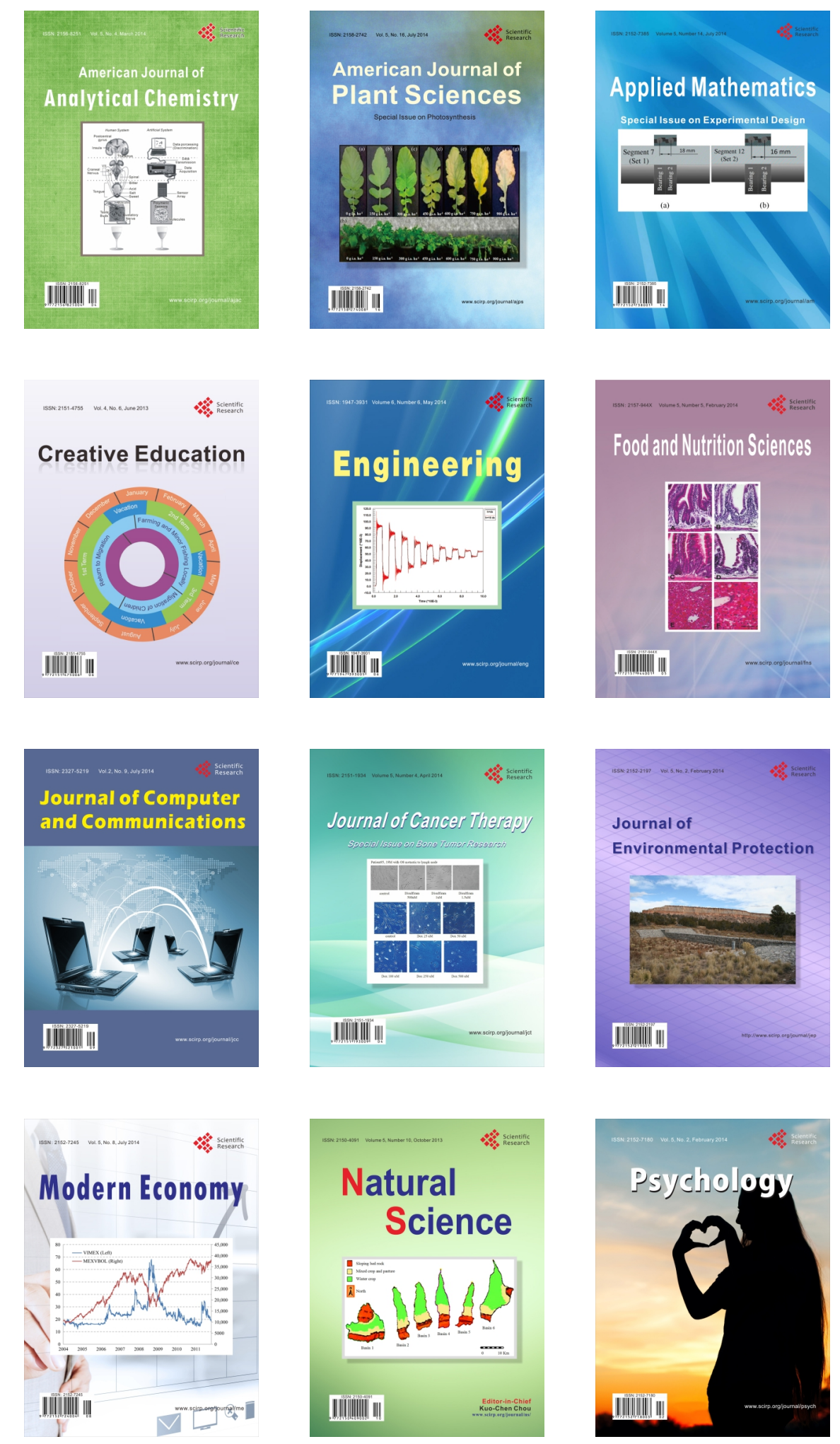